Research Article

\title{
Finite-Time Antisaturation Tracking Control for Hypersonic Vehicle with Uncertain Dynamics
}

\author{
Yuwei Cui $\mathbb{D}^{1,2}$ Yushan $\mathrm{He}^{3}{ }^{3}$ Aijun $\mathrm{Li},{ }^{1}$ Jun $\mathrm{Li},{ }^{3}$ and Shuo Song ${ }^{3}$ \\ ${ }^{1}$ School of Automation, Northwestern Polytechnical University, Xi'an Shaanxi 710072, China \\ ${ }^{2}$ AVIC Xi'an Flight Automatic Control Research Institute, Xi'an Shaanxi 710065, China \\ ${ }^{3}$ School of Automation, Harbin Institute of Technology, Harbin 150001, China \\ Correspondence should be addressed to Yuwei Cui; cuiyuwei0505@mail.nwpu.edu.cn
}

Received 20 August 2020; Revised 10 October 2020; Accepted 24 October 2020; Published 9 November 2020

Academic Editor: Wu Zhonghua

Copyright (c) 2020 Yuwei Cui et al. This is an open access article distributed under the Creative Commons Attribution License, which permits unrestricted use, distribution, and reproduction in any medium, provided the original work is properly cited.

This paper concentrates on the problem of finite-time altitude and velocity tracking control for hypersonic flight vehicles that encounter unmodeled dynamics and input saturations. An adaptive neural finite-time backstepping control strategy is constructed by designing modified virtual commands and compensation signals. The minimum learning parameter algorithm based on a radial basis function is employed to approximate the unknown dynamics with low computational burden. Furthermore, an auxiliary system is established to cope with the nonlinearity caused by actuator saturation. It is concluded by a Lyapunov-based analysis that the finite-time stability is guaranteed under the developed architecture. Finally, numeral simulation is provided to demonstrate the effectiveness of the proposed controller.

\section{Introduction}

In the research of near space aircrafts, generic hypersonic vehicles (GHVs) have become a hotspot in countries all over the world. It owes much to the significant advantages in flight speed and concealment, which makes GHVs possess high application value in both military and civilian fields. However, the unique integrated design and complex flight environment have brought tremendous challenges to the control of GHVs. Facing such a dynamic system with strong coupling, fast time-varying dynamics, high nonlinearity, and uncertainties, scholars have contributed strenuous attempts, including but not limited to sliding mode control [1-3], dynamic compensation control (DCC) [4-6], and adaptive control [7-9].

The backstepping method is an effective strategy to cope with higher-order nonlinear systems, which is widely utilized in trajectory tracking control for GHVs. However, repeated derivation for virtual commands leads to a problem called "explosion of complexity (EOC)." The solution for this obstacle is a dynamic surface control (DSC), in which the low-pass filter is introduced to provide the differential signal of virtual command. In [10], a compound control algorithm based on dynamic compensation was proposed so that the desired trajectory is tracked under strong coupling and high nonlinearity. After that, Zhao and Li designed a robust controller for GHVs with actuator failures, in which the trajectory tracking errors are restricted with a prescribed range [11]. Unfortunately, the above results are not satisfactory enough in terms of convergence speed, which is especially important for a GHV in continual rapid motion. For this reason, an adaptive robust control strategy was constructed for finite-time tracking missions when external disturbances and model uncertainties were encountered [12]. What is more, $\mathrm{Wu}$ et al. established a fixed-time sliding mode backstepping framework, and the time-varying disturbances were estimated via a modified observer [3].

Meanwhile, another ineluctable problem is that the nonlinearity and uncertainty in the GHV dynamics must be paid full attention. As a result, it is difficult to achieve the control objectives through traditional control methods deeply relying on the accurate model information. To relax the conservatism, fuzzy logic control (FLC) [13-15] and neural network (NN) $[1,16,17]$ have become excellent instruments for 
approximation of unknown dynamics. In [13], two fuzzy logic systems were developed to handle the aerodynamic uncertainties of the GHV model. Meanwhile, the constraints of the attitude tracking errors are guaranteed by virtue of the nonlinear mapping method. On the other hand, aiming at the unknown measurement noises, Liu et al. exploited an adaptive fuzzy approximator, which could provide a safeguard for the performances of the proposed controller [14]. Different from the above methodologies, the advantage of the radial basis function neural network (RBFNN) is that it can approximate nonlinear smooth functions with arbitrary precision. Therefore, it is extensively applied in the tracking control of GHV. In [16], adaptive neural networks were employed to tackle unmodeled dynamics, while the unavailable external disturbances were estimated accurately via a novel disturbance observer. To proceed further, Ping et al. formulated a neuroadaptive sliding mode control strategy to eliminate the system uncertainties, where the nonlinearity arising from the actuator failures was alleviated significantly [1].

Besides the capability of antiuncertainty, another important indicator is the stabilization rate. To the best of our knowledge, compared with an asymptotically stable control, the finite-time control performs better in terms of rapidity and robustness. On the basis of a finite time disturbance observer [18], constructs a trajectory tracking backstepping algorithm for a hypersonic flight vehicle with the presence of time-varying environmental disturbance. Subsequently, the finite-time tracking control problem of flexible GHV is investigated in [19], while the unavailable system parameters are taken into consideration. To proceed further, the finitetime tracking control scheme is further extended to the case of unknown actuator failures in [12], where the fixed-time observer is adopted to estimate fault information and other uncertain parameters.

It is noteworthy that another caveat here is input saturation constraint. From a practical point of view, it is impossible for actuators to output infinite control torque due to the restriction of mechanical structure. In [20], an adaptive sliding mode control algorithm was proposed for flexible GHVs, in which adaptive laws were designed to cope with the actuator saturations. Subsequently, Sun et al. constructed a backstepping-based dynamic surface control technique to achieve the altitude and velocity tracking control for GHVs [21]. Not only the phenomenon of EOC is removed by a first-order filter, but also the errors induced by the filter are eliminated via designing compensation signals. Moreover, a specific auxiliary system is developed to conquer input saturation. With further exploration, an adaptive antisaturation controller for GHVs is established in [22], which can ensure that the system still has the capability of stable tracking even in the presence of actuator faults.

Motivated by the above observations, this paper addresses the finite-time trajectory tracking control for GHVs exposed to unmodeled dynamics and input saturations. On the basis of the longitudinal model of GHVs, the altitude and velocity tracking control is achieved via the DCC-based algorithm design. The parameter uncertainties are approximated by RBFNN, and actuator saturations are handled via an auxiliary system. The main contributions of the research are summarized as follows:

(1) Compared with $[10,11]$, the DCC applied in this paper ensures the global finite-time convergence of tracking errors. In addition, the problem of EOC is relaxed, and all the tracking errors and estimation errors will converge to a tiny region containing the origin in finite time

(2) Different from [16], the minimum learning parameter (MLP) algorithm-based RBFNN is employed to approximate the unknown dynamics. In this way, only a single parameter is adaptively updated rather than the whole weight matrix; thus, the excessive occupation of the computational resource is avoided effectively

(3) In consideration of the antisaturation control in [23], the nonlinearity arising from actuator saturation is compensated for effectively, while the auxiliary variable is restricted to a bounded range. In this paper, the hyperbolic tangent function-based modified auxiliary relaxes the conservatism creatively

The remainder of this paper is structured as follows: Section 2 shows the mathematical model and preliminaries; Section 3 details the structure and design procedure of the controller; Section 4 theoretically authenticates the stability of the proposed controller; finally, a simulation example is provided in Section 5 and the conclusion is shown in Section 6.

\section{System Description and Preliminaries}

2.1. Longitudinal Model of GHV. According to the longitudinal model of GHVs in [24], the velocity and attitude dynamic equations are given as

$$
\begin{gathered}
\dot{V}=\frac{T \cos \alpha-D}{m}-\frac{\mu \sin \gamma}{r^{2}} \\
\dot{\gamma}=\frac{L+T \sin \alpha}{m V}-\frac{\left(\mu-\mathrm{V}^{2} \mathrm{r}\right) \cos \gamma}{V r^{2}}, \\
\dot{h}=V \sin \gamma, \\
\dot{\alpha}=Q-\dot{\gamma}, \\
\dot{Q}=\frac{M_{y y}}{I_{y y}},
\end{gathered}
$$

which include five rigid-body states: the velocity $V$, the flightpath angle $\gamma$, the altitude $h$, the angle of attack $\alpha$, and the pitch rate $Q$. Meanwhile, four forces or moments are introduced as the thrust $T$, the $\operatorname{drag} D$, the lift moment $L$, and the pitching moment $M y y$. Other parameters $m, \mu$, and $I_{y y}$ denote the mass, the gravitational constant, and the moment of inertia, respectively. The relevant aerodynamic coefficient is shown as

$$
L=\bar{Q} S C_{L}
$$




$$
\begin{gathered}
D=\bar{Q} S C_{D}, \\
T=\bar{Q} S C_{T}, \\
M_{y y}=\bar{Q} S \bar{c}\left[C_{M}(\alpha)+C_{M}(\delta)+C_{M}(Q)\right], \\
r=h+r_{e}, \\
C_{L}=0.6203 \alpha, \\
C_{D}=0.6450 \alpha^{2}+0.0043378 \alpha+0.003772, \\
C_{T}=\left\{\begin{array}{c}
0.022576 \beta, \\
0.0224+0.00336 \beta, \quad \beta>1, \\
C_{M}(\alpha)=-0.035 \alpha^{2}+0.036117 \alpha+5.3261 \times 10^{-6}, \\
C_{M}(q)=\frac{\bar{c} q\left(-0.6796 \alpha^{2}+0.3015 \alpha-0.2289\right)}{2 V}, \\
C_{M}\left(\delta_{e}\right)=c_{e}\left(\delta_{e}-\alpha\right), \\
c_{e}=0.0292,
\end{array}\right.
\end{gathered}
$$

where the dynamic pressure $\bar{Q}$ satisfies $\bar{Q}=(1 / 2) \rho V^{2}$ with $\rho$ denoting the density of air; $S$ and $r_{e}$ denote the reference area and the radius of the Earth, respectively; and $\bar{c}$ and $c_{e}$ are both constants.

It is noteworthy that most of the existing studies about GHV control lack relevant discussions on actuator saturation. In practice, there is no doubt that the actuator can only output a limited control torque due to the inherent mechanical structure. This paper will provide a solution for tracking control for GHVs in the presence of input saturation constraints and external disturbances. To this end, $\delta_{\mathrm{e}}$ is defined as

$$
\delta_{e}=\operatorname{sat}\left(u_{1}\right)+d_{1}
$$

where $d_{1}$ represents the lumped external disturbance. The saturation nonlinearity $\operatorname{sat}(u)$ is expressed as

$$
\operatorname{sat}(u)= \begin{cases}\operatorname{sign}(u) u_{m}, & u \geq u_{m}, \\ u, & u<u_{m},\end{cases}
$$

where $u_{m}$ denotes the upper bound of sat $(u)$ and $u$ is the desired control input.

2.2. NN-Based Radial Basis Function. In this paper, the unmodeled dynamics of GHVs are approximated via RBFNN. The basic principle of RBFNN is expounded as the following lemma.

Lemma 1 (see [25]). By defining a basis function $\mathbf{H}(\mathbf{x})$, an arbitrary continuous smooth function $f(\mathbf{x})$ can be written in the following form:

$$
f(\mathbf{x})=\mathbf{W}^{* T} \mathbf{H}(\mathbf{x})+o, \quad 0<|o| \leq O
$$

where $\mathbf{x}=\left[x_{1}, x_{2}, \cdots, x_{m}\right]$ represents the input vector and
$\mathbf{W}^{*}=\left[w_{1}, w_{2}, \cdots, w_{p}\right]^{T}$ is the ideal weight matrix; $o$ and $O$ denote the approximation error and its upper bound, respectively; and $\mathbf{h}(\mathbf{x})=\left[h_{1}(\mathbf{x}), h_{2}(\mathbf{x}), \cdots, h_{m}(\mathbf{x})\right]^{T}$ is selected as the Gaussian basis function, whose definition is shown as

$$
h_{i}(\mathbf{x})=\exp \left(-\frac{\left\|\mathbf{x}-\mathbf{c}_{\mathbf{i}}\right\|_{2}^{2}}{2 b_{i}^{2}}\right), \quad i=1, \cdots, p
$$

where $\mathbf{c}_{i} \in \mathbf{R}^{m}$ and $\mathbf{b} \in \mathbf{R}^{p}$ denote the center vector and the width of Gaussian basis function, respectively.

\subsection{Other Preliminaries}

Lemma 2 (see [26]). For $x \in R$ and $\mu>0$, the hyperbolic tangent function possesses the following property:

$$
0<|x|-x \tanh (\mu x) \leq \frac{K}{\mu},
$$

with $K=0.2785$.

Assumption 3. The external disturbances in the GHV system is unknown but bounded, i.e., $0<\left|d_{i}\right| \leq D_{i}, i=1,2$, in which $D_{i}$ are positive constants.

Remark 4. In contrast to the traditional aircraft, GHV mainly works in the near-space area, which possesses the characteristics of complicated environment and dramatically changing aerodynamics. The actual external disturbance torque is usually difficult to observe accurately. Therefore, it is necessary to enhance the controllers' robustness to disturbances and uncertainties.

\section{Finite-Time Controller Design}

In this section, a finite-time tracking controller is developed in virtue of the MLP-based adaptive backstepping strategy. To complete the trajectory tracking control, two subsystems are formulated according to the longitudinal model of GHV in Section 2. For each subsystem, the novel control commands and adaptive laws are designed to ensure the global finite-time stability of a closed-loop system. One caveat here is that the altitude tracking dynamic is a three-order system, in which the effect of EOC is too severe to be neglected. Therefore, the compensation signals are constructed on the basis of a command-filtered control. Meanwhile, a RBFNNbased MLP algorithm is employed to approximate unknown dynamics, such that the burden of computation is relaxed significantly. Input saturations are addressed by resorting to an auxiliary system. All the approximation errors are eliminated along with the unknown external disturbances through the design of the adaptive laws.

3.1. Altitude Control. As the reference trajectory of altitude is denoted by $h_{d}$, the corresponding flight path angle $\gamma_{d}$ can be 
written as

$$
\gamma_{d}=\arcsin \left(\frac{-k_{h} \tilde{h}-k_{h h} \tanh (\tilde{h})+\dot{h}_{d}}{V}\right) \text {, }
$$

where $k_{h}$ and $k_{h h}$ are positive constants and $\gamma_{d} \in\left(-90^{\circ}, 90^{\circ}\right)$.

Considering the GHV model in Section 2, there are three states in the altitude tracking control system, including $\gamma, \vartheta_{p}$, and $q$. Hence, a vector is introduced as

$$
\mathbf{x}=\left[x_{1}, x_{2}, x_{3}\right]^{T}=\left[\gamma, \vartheta_{p}, q\right]^{T},
$$

where $\vartheta_{p}=\alpha+\gamma$. Subsequently, according to Equations (2), (3), (4) and (5), the altitude tracking subsystem of GHV can be expressed as

$$
\left\{\begin{array}{l}
\dot{x}_{1}=f_{1}\left(x_{1}, V\right)+g_{1}(V) x_{2}, \\
\dot{x}_{2}=x_{3} \\
\dot{x}_{3}=f_{3}\left(x_{1}, x_{2}, x_{3}, V\right)+g_{3}(V) \delta_{e}=f_{3}\left(x_{1}, x_{2}, x_{3}, V\right)+g_{3}(V)\left[\operatorname{sat}\left(u_{1}\right)+d_{1}\right],
\end{array}\right.
$$

with

$$
\begin{gathered}
g_{1}(V)=\frac{0.31015 \rho V S}{m}, \\
f_{1}\left(x_{1}, V\right)=\frac{T \sin \alpha}{m V}-\frac{\left(\mu-V^{2} \mathrm{r}\right) \cos \gamma}{V r^{2}}, \\
f_{3}\left(x_{1}, x_{2}, x_{3}, V\right)=\frac{0.5 \rho V^{2} S\left(C_{M}(\alpha)+C_{M}(q)-c_{e} \alpha\right)}{I_{y y}}, \\
g_{3}(V)=\frac{\bar{q} S \bar{c} c_{e}}{I_{y y}} .
\end{gathered}
$$

Assumption 5. The uncertain term $\mathrm{g}_{i}(V)$ possesses an upper bound $\bar{g}_{i}$, which satisfies $0<\left|g_{i}(V)\right| \leq \bar{g}_{i}, i=1,3$.

Remark 6. As pointed above, there exists high uncertainty in the GHV model, due to not only the strong coupling and nonlinearity caused by its own structure but also the fast time variability caused by the high-speed flight. Accordingly, it is difficult to obtain an accurate model for GHVs. In this paper, a RBFNN-based control strategy is proposed to facilitate controller design. Concretely speaking, the functions $f_{i}$ and $g_{i}$ with $i=1,3$ possess unknown structures, which will be approximated by NN. And the resulting approximation error will be eliminated by resorting to the design of adaptive laws.
With the introduction of reference trajectory of states, the tracking errors are defined as

$$
\begin{gathered}
\varepsilon_{1}=x_{1}-x_{1 d}, \\
\varepsilon_{2}=x_{2}-x_{2 d}, \\
\varepsilon_{3}=x_{3}-x_{3 d}-\xi_{1} \tanh \left(\chi_{1}\right),
\end{gathered}
$$

where

$$
\dot{\chi}_{1}=\frac{\cosh ^{2} \chi_{1}}{\xi_{1}}\left[-\zeta_{1} \tanh \left(\chi_{1}\right)+\operatorname{sat}\left(u_{1}\right)-u_{1}\right], \quad \chi_{1}(0)=0
$$

with the designed parameters satisfying $\xi_{1}, \zeta_{1}>0, x_{i d}$ being the designed trajectory.

Remark 7. In [23], the auxiliary system and the transformed error are defined as $\dot{\chi}=-\zeta \chi+\operatorname{sat}(u)-u$ and $\varepsilon=x-x_{d}-\xi \chi$ , respectively. However, this solution is effective only if the auxiliary variable $\chi$ is bounded, while the dynamics (27) and (28) employs the hyperbolic tangent function to construct a bounded compensation term $\zeta \tanh (\chi)$, so that the conservatism of above assumption is relaxed.

Taking the time derivative of equations and substituting Equation (25) yields

$$
\begin{gathered}
\dot{\varepsilon}_{1}=f_{1}+g_{1} x_{2}-\dot{x}_{1 d}, \\
\dot{\varepsilon}_{2}=x_{3}-\dot{x}_{2 d}, \\
\dot{\varepsilon}_{3}=f_{3}+g_{3}\left[\operatorname{sat}\left(u_{1}\right)+d_{1}\right]-\dot{x}_{3 \mathrm{~d}}+\zeta_{1} \tanh \left(\chi_{1}\right)-\operatorname{sat}\left(u_{1}\right)+u_{1} .
\end{gathered}
$$

For the purpose of relaxing the issue of EOC, a first-order low-pass filter is introduced as

$$
\begin{array}{ll}
c_{2} \dot{x}_{2 d}+x_{2 d}=x_{2 c}, & x_{2 d}(0)=0, \\
c_{3} \dot{x}_{3 d}+x_{3 d}=x_{3 c}, & x_{3 d}(0)=0,
\end{array}
$$

where $x_{2 c}, x_{3 c}$ are both output signals and the filter parameters satisfy $c_{2}, c_{3}>0$. Resulting error variables are defined as

$$
y_{2}=x_{2 d}-x_{2 c}, y_{3}=x_{3 d}-x_{3 c}
$$

Step 1. Taking the unavailable dynamic $f_{1}$ into account and using Lemma 2 yield

$$
\begin{gathered}
f_{1}=\mathbf{W}_{1}^{* T} \mathbf{H}_{1}(\mathbf{x})+o_{1}, \quad 0<\left|o_{1}\right| \leq O_{1}, \\
\left\|\mathbf{W}_{1}^{* T} \mathbf{H}_{1}\right\| \leq\left\|\mathbf{W}_{1}^{* T}\right\|\left\|\mathbf{H}_{1}\right\| \leq w_{1} h_{1},
\end{gathered}
$$

where $h_{1}=\left\|\mathbf{H}_{1}\right\|$ and $w_{1} \geq\left\|\mathbf{W}_{1}^{* T}\right\|$. 
The compensation signal is designed as

$$
\dot{\varphi}_{1}=-\vartheta_{1} \varphi_{1}-\vartheta_{2} \tanh \left(\varphi_{1}\right)-\frac{\left|g_{1} \varepsilon_{1} y_{2}\right|+0.5 y_{2}^{2}}{\varphi_{1}}+y_{2} \text {, }
$$

where $\vartheta_{1}, \vartheta_{2}$ are both positive constants.

Subsequently, the adaptive laws and the virtual command are designed as

$$
\begin{gathered}
\dot{\widehat{w}}_{1}=\alpha_{1}\left(\left|\varepsilon_{1}\right| h_{1}-r_{1} \widehat{w}_{1}\right), \\
\dot{\widehat{R}}_{1}=\beta_{1}\left(\left|\varepsilon_{1}\right|-r_{2} \widehat{R}_{1}\right), \\
x_{2 c}=\frac{1}{g_{1}}\left[\dot{x}_{1 d}-k_{1} \varepsilon_{1}-k_{2} \tanh \left(\varepsilon_{1}\right)-\widehat{w}_{1} h_{1} \tanh \left(\varepsilon_{1}\right)\right. \\
\left.-\widehat{R}_{1} \tanh \left(\varepsilon_{1}\right)+l_{1} \varphi_{1}\right],
\end{gathered}
$$

in which $R_{1}=O_{1} ; \widehat{w}_{1}$ and $\widehat{R}_{1}$ denote estimations of $w_{1}$ and $R_{1}$, respectively; and parameters $\alpha_{1}, \beta_{1}, r_{1}, r_{2}, k_{1}, k_{2}, l_{1}$ are all designed as positive constants.

Remark 8. Different from the conventional algorithm of RBFNN, MLP algorithms select the upper bound of the weight matrix norm as the online updating parameter. Hence, only a single parameter is estimated rather than the whole weight matrix, so that the excessive occupation of the computational resource is relaxed significantly.

To show the stability of $\varepsilon_{1}$, the Lyapunov function (LF) is chosen as

$$
F_{1}=\frac{1}{2} \varepsilon_{1}^{2}+\frac{1}{2} \varphi_{1}^{2}
$$
yield

Differentiating $F_{1}$ and using Equations (33) and (40)

$$
\begin{aligned}
\dot{F}_{1}= & \varepsilon_{1} \dot{\varepsilon}_{1}+\varphi_{1} \dot{\varphi}_{1}=\varepsilon_{1}\left[f_{1}+g_{1}\left(\varepsilon_{2}+x_{2 d}\right)-\dot{x}_{1 d}\right]+\varphi_{1} \\
& \cdot\left[-\vartheta_{1} \varphi_{1}-\vartheta_{2} \tanh \left(\varphi_{1}\right)-\frac{\left|g_{1} \varepsilon_{1} y_{2}\right|+0.5 y_{2}^{2}}{\varphi_{1}}+y_{2}\right] \\
= & \varepsilon_{1} f_{1}+g_{1} \varepsilon_{1} \varepsilon_{2}+g_{1} \varepsilon_{1} y_{2}+\varepsilon_{1} \\
& \cdot\left[-k_{1} \varepsilon_{1}-k_{2} \tanh \left(\varepsilon_{1}\right)-\widehat{w}_{1} h_{1} \tanh \left(\varepsilon_{1}\right)-\widehat{R}_{1} \tanh \left(\varepsilon_{1}\right)+l_{1} \varphi_{1}\right] \\
& -\vartheta_{1} \varphi_{1}^{2}-\vartheta_{2} \varphi_{1} \tanh \left(\varphi_{1}\right)-\left|g_{1} \varepsilon_{1} y_{2}\right|-\frac{1}{2} y_{2}^{2}+\varphi_{1} y_{2} \leq \varepsilon_{1} f_{1} \\
& +g_{1} \varepsilon_{1} \varepsilon_{2}-k_{1} \varepsilon_{1}^{2}-k_{2} \varepsilon_{1} \tanh \left(\varepsilon_{1}\right)-\widehat{w}_{1} h_{1} \varepsilon_{1} \tanh \left(\varepsilon_{1}\right) \\
& -\widehat{R}_{1} \varepsilon_{1} \tanh \left(\varepsilon_{1}\right)+\varepsilon_{1}^{2}+\frac{1}{4} l_{1}^{2} \varphi_{1}^{2}-\vartheta_{1} \varphi_{1}^{2}-\vartheta_{2} \varphi_{1} \tanh \left(\varphi_{1}\right)+\frac{1}{2} \varphi_{1}^{2} .
\end{aligned}
$$

According to Lemma 2, one has

$$
\begin{gathered}
-k_{2} \varepsilon_{1} \tanh \left(\varepsilon_{1}\right) \leq-k_{2}\left|\varepsilon_{1}\right|+k_{2} \omega, \\
-\widehat{w}_{1} h_{1} \varepsilon_{1} \tanh \left(\varepsilon_{1}\right) \leq-\widehat{w}_{1} h_{1}\left|\varepsilon_{1}\right|+\widehat{w}_{1} h_{1} \omega, \\
-\widehat{R}_{1} \varepsilon_{1} \tanh \left(\varepsilon_{1}\right) \leq-\widehat{R}_{1}\left|\varepsilon_{1}\right|+\widehat{R}_{1} \omega, \\
-\vartheta_{2} \varphi_{1} \tanh \left(\varphi_{1}\right) \leq-\vartheta_{2}\left|\varphi_{1}\right|+\vartheta_{2} \omega .
\end{gathered}
$$

Substituting Equation (39) into Equation (38) leads to

$$
\begin{aligned}
\dot{F}_{1} \leq & \varepsilon_{1}\left(\mathbf{W}_{1}^{* T} \mathbf{H}_{1}(\mathbf{x})+o_{1}\right)+g_{1} \varepsilon_{1} \varepsilon_{2}-\left(k_{1}-1\right) \varepsilon_{1}^{2}-k_{2}\left|\varepsilon_{1}\right|+k_{2} \omega \\
& -\widehat{w}_{1} h_{1}\left|\varepsilon_{1}\right|+\widehat{w}_{1} h_{1} \omega-\widehat{R}_{1}\left|\varepsilon_{1}\right|+\widehat{R}_{1} \omega-\left(\vartheta_{1}-\frac{1}{4} l_{1}^{2}-1\right) \varphi_{1}^{2} \\
& -\vartheta_{2}\left|\varphi_{1}\right|+\vartheta_{2} \omega \leq\left|\varepsilon_{1}\right| w_{1} h_{1}+\left|\varepsilon_{1}\right| R_{1}+g_{1} \varepsilon_{1} \varepsilon_{2}-\left(k_{1}-1\right) \varepsilon_{1}^{2} \\
& -k_{2}\left|\varepsilon_{1}\right|+k_{2} \omega-\widehat{w}_{1} h_{1}\left|\varepsilon_{1}\right|+\frac{h_{1}^{2} \widehat{w}_{1}^{2}}{2}+\frac{\omega^{2}}{2}-\widehat{R}_{1}\left|\varepsilon_{1}\right|+\frac{\widehat{R}_{1}^{2}}{2} \\
& +\frac{\omega^{2}}{2}-\left(\vartheta_{1}-\frac{1}{4} l_{1}^{2}-\frac{1}{2}\right) \varphi_{1}^{2}-\vartheta_{2}\left|\varphi_{1}\right|+\vartheta_{2} \omega=\left|\varepsilon_{1}\right| \tilde{w}_{1} h_{1} \\
& +\left|\varepsilon_{1}\right| \tilde{R}_{1}-\left(k_{1}-1-\frac{1}{2} \bar{g}_{1}\right) \varepsilon_{1}^{2}+\frac{1}{2} \bar{g}_{1} \varepsilon_{2}^{2}-k_{2}\left|\varepsilon_{1}\right|- \\
& +\left(\vartheta_{1}-\frac{1}{4} l_{1}^{2}-\frac{1}{2}\right) \varphi_{1}^{2}-\vartheta_{2}\left|\varphi_{1}\right|+\frac{h_{1}^{2} \widehat{w}_{1}^{2}}{2}+\frac{\widehat{R}_{1}^{2}}{2}+\omega^{2} \\
& +k_{2} \omega+\vartheta_{2} \omega .
\end{aligned}
$$

Step 2. For the purpose of the stabilization of $\varepsilon_{2}$, the virtual command $x_{3 d}$ is given as

$$
x_{3 c}=-k_{3} \varepsilon_{2}-k_{4} \varepsilon_{2} \tanh \left(\varepsilon_{2}\right)+\dot{x}_{2 d}+l_{2} \varphi_{2},
$$

where $k_{3}, k_{4}$, and $l_{2}$ are all positive parameters.

The filter compensation signal is expressed as

$$
\dot{\varphi}_{2}=-\vartheta_{3} \varphi_{2}-\vartheta_{4} \tanh \left(\varphi_{2}\right)-\frac{\left|\varepsilon_{2} y_{3}\right|+0.5 y_{3}^{2}}{\varphi_{2}}+y_{3} \text {, }
$$

where $\vartheta_{3}, \vartheta_{4}$ are both positive parameters.

The second LF is selected, and its derivatives are presented as

$$
\begin{gathered}
F_{2}=\frac{1}{2} \varepsilon_{2}^{2}+\frac{1}{2} \varphi_{2}^{2}, \\
\dot{F}_{2}=\varepsilon_{2} \dot{\varepsilon}_{2}+\varphi_{2} \dot{\varphi}_{2}=\varepsilon_{2}\left(\varepsilon_{3}+x_{3 d}-\dot{x}_{2 d}\right)+\varphi_{2} \\
\cdot\left(-\vartheta_{3} \varphi_{2}-\vartheta_{4} \tanh \left(\varphi_{2}\right)-\frac{1}{2 \varphi_{2}} y_{3}^{2}+y_{3}\right) \\
=\varepsilon_{2} \varepsilon_{3}+\varepsilon_{2} y_{3}-k_{3} \varepsilon_{2}^{2}-k_{4} \varepsilon_{2} \tanh \left(\varepsilon_{2}\right)-g_{1} \varepsilon_{1} \varepsilon_{2}+\varepsilon_{2} l_{2} \varphi_{2} \\
-\vartheta_{3} \varphi_{2}^{2}-\vartheta_{4} \varphi_{2} \tanh \left(\varphi_{2}\right)-\left|\varepsilon_{2} y_{3}\right|-\frac{1}{2} y_{3}^{2}+\varphi_{2} y_{3} \leq \varepsilon_{2} \varepsilon_{3} \\
-k_{3} \varepsilon_{2}^{2}-k_{4} \varepsilon_{2} \tanh \left(\varepsilon_{2}\right)-g_{1} \varepsilon_{1} \varepsilon_{2}+\varepsilon_{2}^{2}+\frac{1}{4} l_{2}^{2} \varphi_{2}^{2}-\vartheta_{3} \varphi_{2}^{2} \\
-\vartheta_{4} \varphi_{2} \tanh \left(\varphi_{2}\right)+\frac{1}{2} \varphi_{2}^{2} .
\end{gathered}
$$

It can be derived from Lemma 2 that

$$
\begin{aligned}
& -k_{4} \varepsilon_{1} \tanh \left(\varepsilon_{2}\right) \leq-k_{4}\left|\varepsilon_{2}\right|+k_{4} \omega, \\
& -\vartheta_{4} \varphi_{2} \tanh \left(\varphi_{2}\right) \leq-\vartheta_{4}\left|\varphi_{2}\right|+\vartheta_{4} \omega .
\end{aligned}
$$


Substituting the result into Equation (44), it obtains

$$
\begin{gathered}
\dot{F}_{2} \leq \varepsilon_{2} \varepsilon_{3}-\left(k_{3}-1-\frac{1}{2} \bar{g}_{1}\right) \varepsilon_{2}^{2}+\frac{1}{2} \bar{g}_{1} \varepsilon_{1}^{2}-k_{4}\left|\varepsilon_{2}\right|+k_{4} \omega- \\
\cdot\left(\vartheta_{3}-\frac{1}{4} l_{2}^{2}-\frac{1}{2}\right) \varphi_{2}^{2}-\vartheta_{4}\left|\varphi_{2}\right|+\vartheta_{4} \omega
\end{gathered}
$$

Step 3. In this part, the uncertain dynamic $f_{3}$ is approximated via $\mathrm{RBFNN}$, which is shown as

$$
\begin{aligned}
& f_{3}=\mathbf{W}_{3}^{* T} \mathbf{H}_{3}(\mathbf{x})+o_{3}, \quad 0<\left|o_{3}\right| \leq O_{3}, \\
& \left\|\mathbf{W}_{3}^{* T} \mathbf{H}_{3}\right\| \leq\left\|\mathbf{W}_{3}^{* T}\right\|\left\|\mathbf{H}_{3}\right\| \leq w_{3} h_{3},
\end{aligned}
$$

where $h_{3}=\left\|\mathbf{H}_{3}\right\|$ and $w_{3} \geq\left\|\mathbf{W}_{3}^{* T}\right\|$.

Owing to the effect of external disturbances, it is defined according to Assumptions 3 and 5 that $R_{3}=\bar{g}_{3}\left[u_{m}+D_{1}\right]+$ $\mathrm{O}_{3}+\zeta_{1}-u_{m}$. Afterward, the adaptive laws and the control input are given as

$$
\begin{gathered}
\dot{\hat{w}}_{3}=\alpha_{3}\left(\left|\varepsilon_{3}\right| h_{3}-r_{3} \widehat{w}_{3}\right), \\
\dot{\hat{R}}_{3}=\beta_{3}\left(\left|\varepsilon_{3}\right|-r_{4} \widehat{R}_{3}\right) \\
u_{1}=\frac{1}{g_{3}}\left(-k_{5} \varepsilon_{3}-\frac{k_{6} \varepsilon_{3}}{\left|\varepsilon_{3}\right|+\rho_{1}}-\frac{\widehat{w}_{3} h_{3} \varepsilon_{3}}{\left|\varepsilon_{3}\right|+\rho_{2}}-\frac{\widehat{R}_{3} \varepsilon_{3}}{\left|\varepsilon_{3}\right|+\rho_{3}}+\dot{x}_{3 d}\right), \\
\rho_{1}=\frac{b_{1}}{1+k_{6}}, \rho_{2}=\frac{b_{2}}{1+\widehat{w}_{3} h_{3}}, \rho_{3}=\frac{b_{3}}{1+\widehat{R}_{3}},
\end{gathered}
$$

where parameters $\alpha_{3}, \beta_{3}, r_{3}, r_{4}, k_{5}, k_{6}, \rho_{1}, \rho_{2}, \rho_{3}$ are all positive constants.

Remark 9. Considering the controller (51), several ingredients should to be emphasized as follows: (i) The term $-k_{5} \varepsilon_{3}$ and the term $-\left(k_{6} \varepsilon_{3} /\left(\left|\varepsilon_{3}\right|+\rho_{1}\right)\right)$ guarantee the finite time convergence of $\varepsilon_{3}$. Meanwhile, the term $-\left(\widehat{w}_{3} h_{3} \varepsilon_{3} /\left(\left|\varepsilon_{3}\right|+\rho_{2}\right.\right.$ )) with the construction (49) is utilized to estimate the weight parameter of $\mathrm{NN}$, and the term $-\left(\widehat{R}_{3} \varepsilon_{3} /\left(\left|\varepsilon_{3}\right|+\rho_{3}\right)\right)$ is for unknown lumped disturbances. (ii) The introduction of variables $\varepsilon_{i}(i=1,2,3)$ not only leads to the attenuation of chattering phenomenon but also improves the controllability of steady-state errors. This point will be proved in subsequent derivations.

Consequently, the third LF and its derivative are presented as

$$
F_{3}=\frac{1}{2} \varepsilon_{3}^{2}
$$

$\dot{F}_{3}=\varepsilon_{3} \dot{\varepsilon}_{3}=\varepsilon_{3}\left[f_{3}+g_{3}\left[\operatorname{sat}\left(u_{1}\right)+d_{1}\right]-\dot{x}_{3 \mathrm{~d}}+\zeta_{1} \tanh \left(\chi_{1}\right)\right.$ $\left.-\operatorname{sat}\left(u_{1}\right)+u_{1}\right]=\varepsilon_{3} f_{3}-k_{5} \varepsilon_{3}^{2}-k_{6} \varepsilon_{3}^{2} /\left|\varepsilon_{3}\right|+\rho_{1}-\widehat{w}_{3} h_{3} \varepsilon_{3}^{2} /\left|\varepsilon_{3}\right|+$ $\rho_{2}-\widehat{R}_{3} \varepsilon_{3}^{2} /\left|\varepsilon_{3}\right|+\rho_{3}-\varepsilon_{2} \varepsilon_{3}+\varepsilon_{3}\left[g_{3}\left[\operatorname{sat}\left(u_{1}\right)+d_{1}\right]+\zeta_{1} \tanh \left(\chi_{1}\right.\right.$ )$\left.-\operatorname{sat}\left(u_{1}\right)\right]$.
Taking Equations (47) and (48) into account, it leads to

$$
\begin{aligned}
\dot{F}_{3}= & \varepsilon_{3}\left(\mathbf{W}_{3}^{* T} \mathbf{H}_{3}(\mathbf{x})+o_{3}\right)-k_{5} \varepsilon_{3}^{2}-\frac{k_{6} \varepsilon_{3}^{2}}{\left|\varepsilon_{3}\right|+\rho_{1}}-\frac{\widehat{w}_{3} h_{3} \varepsilon_{3}^{2}}{\left|\varepsilon_{3}\right|+\rho_{2}} \\
& -\frac{\widehat{R}_{3} \varepsilon_{3}^{2}}{\left|\varepsilon_{3}\right|+\rho_{3}}-\varepsilon_{2} \varepsilon_{3}+\varepsilon_{3}\left[g_{3}\left[\operatorname{sat}\left(u_{1}\right)+d_{1}\right]+\zeta_{1} \tanh \left(\chi_{1}\right)\right. \\
& \left.-\operatorname{sat}\left(u_{1}\right)\right] \leq\left|\varepsilon_{3}\right| w_{3} h_{3}-k_{5} \varepsilon_{3}^{2}-\frac{k_{6} \varepsilon_{3}^{2}}{\left|\varepsilon_{3}\right|+\rho_{1}}-\frac{\widehat{w}_{3} h_{3} \varepsilon_{3}^{2}}{\left|\varepsilon_{3}\right|+\rho_{2}} \\
& -\frac{\widehat{R}_{3} \varepsilon_{3}^{2}}{\left|\varepsilon_{3}\right|+\rho_{3}}-\varepsilon_{2} \varepsilon_{3}+\varepsilon_{3}\left[g_{3}\left[\operatorname{sat}\left(u_{1}\right)+d_{1}\right]+O_{3}\right. \\
& \left.+\zeta_{1} \tanh \left(\chi_{1}\right)-\operatorname{sat}\left(u_{1}\right)\right] \leq\left|\varepsilon_{3}\right| w_{3} h_{3}-k_{5} \varepsilon_{3}^{2}-\frac{k_{6} \varepsilon_{3}^{2}}{\left|\varepsilon_{3}\right|+\rho_{1}} \\
& -\frac{\widehat{w}_{3} h_{3} \varepsilon_{3}^{2}}{\left|\varepsilon_{3}\right|+\rho_{2}}-\frac{\widehat{R}_{3} \varepsilon_{3}^{2}}{\left|\varepsilon_{3}\right|+\rho_{3}}-\varepsilon_{2} \varepsilon_{3}+\left|\varepsilon_{3}\right| R_{3} .
\end{aligned}
$$

Some of the items in Equation (54) are scaled as

$$
\begin{gathered}
-\frac{k_{6} \varepsilon_{3}^{2}}{\left|\varepsilon_{3}\right|+\rho_{1}}=-k_{6}\left|\varepsilon_{3}\right|+k_{6} \rho_{1} \times \frac{\left|\varepsilon_{3}\right|}{\left|\varepsilon_{3}\right|+\rho_{1}} \\
=-k_{6}\left|\varepsilon_{3}\right|+\frac{k_{6} b_{1}}{1+k_{6}} \times \frac{\left|\varepsilon_{3}\right|}{\left|\varepsilon_{3}\right|+\rho_{1}} \leq-k_{6}\left|\varepsilon_{3}\right|+b_{1} \\
-\frac{\widehat{w}_{3} h_{3} \varepsilon_{3}^{2}}{\left|\varepsilon_{3}\right|+\rho_{2}}=-\widehat{w}_{3} h_{3}\left|\varepsilon_{3}\right|+\widehat{w}_{3} h_{3} \rho_{2} \times \frac{\left|\varepsilon_{3}\right|}{\left|\varepsilon_{3}\right|+\rho_{2}} \\
=-\widehat{w}_{3} h_{3}\left|\varepsilon_{3}\right|+\frac{\widehat{w}_{3} h_{3} b_{2}}{1+\widehat{w}_{3} h_{3}} \times \frac{\left|\varepsilon_{3}\right|}{\left|\varepsilon_{3}\right|+\rho_{2}} \\
\leq-\widehat{w}_{3} h_{3}\left|\varepsilon_{3}\right|+b_{2}, \\
-\frac{\widehat{R}_{3} \varepsilon_{3}^{2}}{\left|\varepsilon_{3}\right|+\rho_{3}}=-\widehat{R}_{3}\left|\varepsilon_{3}\right|+\widehat{R}_{3} \rho_{3} \times \frac{\left|\varepsilon_{3}\right|}{\left|\varepsilon_{3}\right|+\rho_{3}} \\
=-\widehat{R}_{3}\left|\varepsilon_{3}\right|+\frac{\widehat{R}_{3} b_{3}}{1+\widehat{R}_{3}} \times \frac{\left|\varepsilon_{3}\right|}{\left|\varepsilon_{3}\right|+\rho_{3}} \leq-\widehat{R}_{3}\left|\varepsilon_{3}\right|+b_{3} .
\end{gathered}
$$

With the substitution of these results, Equation (54) becomes

$$
\begin{aligned}
\dot{F}_{3} \leq & \left|\varepsilon_{3}\right| w_{3} h_{3}-k_{5} \varepsilon_{3}^{2}-k_{6}\left|\varepsilon_{3}\right|+b_{1}-\widehat{w}_{3} h_{3}\left|\varepsilon_{3}\right|+b_{2}-\widehat{R}_{1}\left|\varepsilon_{3}\right| \\
& +b_{3}-\varepsilon_{2} \varepsilon_{3}+\left|\varepsilon_{3}\right| R_{3} \leq\left|\varepsilon_{3}\right| \tilde{w}_{3} h_{3}+\left|\varepsilon_{3}\right| \tilde{R}_{3}-\left(k_{5}-\frac{1}{2}\right) \varepsilon_{3}^{2} \\
& +\frac{1}{2} \varepsilon_{2}^{2}-k_{6}\left|\varepsilon_{3}\right|+b_{1}+b_{2}+b_{3} .
\end{aligned}
$$

3.2. Velocity Control. As shown in Section 2, the closed-loop system for velocity tracking control is expressed as

$$
\dot{V}=f_{v}+\operatorname{sat}\left(u_{2}\right)+d_{2} \text {, }
$$


with

$$
f_{v}=\frac{[T \cos (\theta-\gamma)-D]}{m}-g \sin \gamma-u_{2}
$$

According to Lemma 2, the unavailable state $f_{v}$ can be written as

$$
\begin{gathered}
f_{v}=\mathbf{W}_{v}^{* T} \mathbf{H}_{v}(\mathbf{x})+o_{v}, \quad 0<o_{v} \leq O_{v}, \\
\left\|\mathbf{W}_{v}^{* T} \mathbf{H}_{v}\right\| \leq\left\|\mathbf{W}_{v}^{* T}\right\|\left\|\mathbf{H}_{v}\right\| \leq w_{v} h_{v},
\end{gathered}
$$

where $h_{v}=\left\|\mathbf{H}_{v}\right\|$ and $w_{v} \geq\left\|\mathbf{W}_{v}^{* T}\right\|$.

The velocity tracking error $e_{v}$ is introduced as

$$
\varepsilon_{v}=V-V_{d}-\zeta_{2} \tanh \left(\chi_{2}\right),
$$

where

$$
\dot{\chi}_{2}=\frac{\cosh ^{2} \chi_{2}}{\xi_{2}}\left[-\zeta_{2} \tanh \left(\chi_{2}\right)+\operatorname{sat}\left(u_{2}\right)-u_{2}\right], \quad \chi_{2}(0)=0,
$$

with the parameters satisfying $\xi_{2}, \zeta_{2}>0$.

Differentiating $\varepsilon_{v}$ along Equation (61) and using Equation (57) yield

$$
\dot{\varepsilon}_{v}=f_{v}+d_{2}-\dot{V}_{d}+\zeta_{2} \tanh \left(\chi_{2}\right)+u_{2} .
$$

It is defined that $R_{v}=D_{2}+O_{v}+\zeta_{1}$. Subsequently, the adaptive laws and the control input are given as

$$
\begin{gathered}
\dot{\hat{w}}_{v}=\alpha_{v}\left(\left|\varepsilon_{v}\right| h_{v}-r_{5} \widehat{w}_{v}\right), \\
\dot{\hat{R}}_{v}=\beta_{v}\left(\left|\varepsilon_{v}\right|-r_{6} \widehat{R}_{v}\right), \\
u_{2}=-k_{7} \varepsilon_{v}-\frac{k_{8} \varepsilon_{v}}{\left|\varepsilon_{v}\right|+\rho_{4}}-\frac{\widehat{w}_{v} h_{v} \varepsilon_{v}}{\left|\varepsilon_{v}\right|+\rho_{5}}-\frac{\widehat{R}_{v} \varepsilon_{v}}{\left|\varepsilon_{v}\right|+\rho_{6}}-\dot{V}_{d}, \\
\rho_{4}=\frac{b_{4}}{1+k_{8}}, \rho_{5}=\frac{b_{5}}{1+\widehat{w}_{v} h_{v}}, \rho_{6}=\frac{b_{6}}{1+\widehat{R}_{v}},
\end{gathered}
$$

where the parameters $\alpha_{v}, \beta_{v}, r_{5}, r_{6}, k_{7}, k_{8}, b_{4}, b_{5}, b_{6}$ are all designed as positive constants.

To illustrate the convergence of the velocity tracking error, the LF is selected as

$$
F_{v}=\frac{1}{2} \varepsilon_{v}^{2}
$$

Differentiating $F_{v}$ along $(78)$ yields

$$
\begin{aligned}
\dot{F}_{v}= & \varepsilon_{v} \dot{\varepsilon}_{v}=\varepsilon_{v}\left[f_{v}+d_{2}-\dot{V}_{d}+\zeta_{2} \tanh \left(\chi_{2}\right)+u_{2}\right] \\
= & \varepsilon_{v} f_{v}-k_{7} \varepsilon_{v}^{2}-\frac{k_{8} \varepsilon_{v}^{2}}{\left|\varepsilon_{v}\right|+\rho_{4}}-\frac{\widehat{w}_{v} h_{v} \varepsilon_{v}^{2}}{\left|\varepsilon_{v}\right|+\rho_{5}}-\frac{\widehat{R}_{v} \varepsilon_{v}^{2}}{\left|\varepsilon_{v}\right|+\rho_{6}} \\
& +\varepsilon_{v}\left[d_{2}+\zeta_{2} \tanh \left(\chi_{2}\right)\right] .
\end{aligned}
$$

TABLE 1: Initial values of states.

\begin{tabular}{lcc}
\hline Item & Value & $\mathrm{Unit}$ \\
\hline$V$ & 15060 & $\mathrm{ft} / \mathrm{s}$ \\
$h$ & 110000 & $\mathrm{ft}$ \\
$\gamma$ & 0 & $\mathrm{rad}$ \\
$\alpha$ & $1.6325 \pi / 180$ & $\mathrm{rad}$ \\
$q$ & 0 & $\mathrm{rad} / \mathrm{s}$ \\
\hline
\end{tabular}

With the substitution of Equations (59) and (60), it is further derived as

$$
\begin{aligned}
\dot{F}_{v}= & \varepsilon_{v}\left(\mathbf{W}_{v}^{* T} \mathbf{H}_{v}(\mathbf{x})+o_{v}\right)-k_{7} \varepsilon_{v}^{2}-\frac{k_{8} \varepsilon_{v}^{2}}{\left|\varepsilon_{v}\right|+\rho_{4}}-\frac{\widehat{w}_{v} h_{v} \varepsilon_{v}^{2}}{\left|\varepsilon_{v}\right|+\rho_{5}} \\
& -\frac{\widehat{R}_{v} \varepsilon_{v}^{2}}{\left|\varepsilon_{v}\right|+\rho_{6}}+\varepsilon_{v}\left[d_{2}+\zeta_{2} \tanh \left(\chi_{2}\right)\right] \leq\left|\varepsilon_{v}\right| w_{v} h_{v}-k_{7} \varepsilon_{v}^{2} \\
& -\frac{k_{8} \varepsilon_{v}^{2}}{\left|\varepsilon_{v}\right|+\rho_{4}}-\frac{\widehat{w}_{v} h_{v} \varepsilon_{v}^{2}}{\left|\varepsilon_{v}\right|+\rho_{5}}-\frac{\widehat{R}_{v} \varepsilon_{v}^{2}}{\left|\varepsilon_{v}\right|+\rho_{6}}+\varepsilon_{v}\left[d_{2}+O_{v}\right. \\
& \left.+\zeta_{2} \tanh \left(\chi_{2}\right)\right] \leq\left|\varepsilon_{v}\right| w_{v} h_{v}-k_{7} \varepsilon_{v}^{2}-\frac{k_{8} \varepsilon_{v}^{2}}{\left|\varepsilon_{v}\right|+\rho_{4}}-\frac{\widehat{w}_{v} h_{v} \varepsilon_{v}^{2}}{\left|\varepsilon_{v}\right|+\rho_{5}} \\
& -\frac{\widehat{R}_{v} \varepsilon_{v}^{2}}{\left|\varepsilon_{v}\right|+\rho_{6}}+\left|\varepsilon_{v}\right| R_{v} .
\end{aligned}
$$

There holds the inequations that

$$
\begin{aligned}
-\frac{k_{8} \varepsilon_{v}^{2}}{\left|\varepsilon_{v}\right|+\rho_{4}}= & -k_{8}\left|\varepsilon_{v}\right|+\left(k_{8} \rho_{4}\right) \frac{\left|\varepsilon_{v}\right|}{\left|\varepsilon_{v}\right|+\rho_{4}}=-k_{8}\left|\varepsilon_{v}\right|+\frac{k_{8} b_{4}}{1+k_{8}} \\
& \times \frac{\left|\varepsilon_{v}\right|}{\left|\varepsilon_{v}\right|+\rho_{4}} \leq-k_{8}\left|\varepsilon_{v}\right|+b_{4},
\end{aligned}
$$

$$
\begin{aligned}
-\frac{\widehat{w}_{v} h_{v} \varepsilon_{v}^{2}}{\left|\varepsilon_{v}\right|+\rho_{5}}= & -\widehat{w}_{v} h_{v}\left|\varepsilon_{v}\right|+\left(\widehat{w}_{v} h_{v} \rho_{5}\right) \frac{\left|\varepsilon_{v}\right|}{\left|\varepsilon_{v}\right|+\rho_{5}}=-\widehat{w}_{v} h_{v}\left|\varepsilon_{v}\right| \\
& +\frac{\widehat{w}_{v} h_{v} b_{5}}{1+\widehat{w}_{v} h_{v}} \times \frac{\left|\varepsilon_{v}\right|}{\left|\varepsilon_{v}\right|+\rho_{5}} \leq-\widehat{w}_{v} h_{v}\left|\varepsilon_{v}\right|+b_{5},
\end{aligned}
$$

$$
\begin{aligned}
-\frac{\widehat{R}_{v} \varepsilon_{v}^{2}}{\left|\varepsilon_{v}\right|+\rho_{6}}= & -\widehat{R}_{v}\left|\varepsilon_{v}\right|+\left(\widehat{R}_{v} \rho_{6}\right) \frac{\left|\varepsilon_{v}\right|}{\left|\varepsilon_{v}\right|+\rho_{6}}=-\widehat{R}_{v}\left|\varepsilon_{v}\right|+\frac{\widehat{R}_{v} b_{6}}{1+\widehat{R}_{v}} \\
& \times \frac{\left|\varepsilon_{v}\right|}{\left|\varepsilon_{v}\right|+\rho_{6}} \leq-\widehat{R}_{v}\left|\varepsilon_{v}\right|+b_{6} .
\end{aligned}
$$

Substituting the results into Equation (70), one obtains

$$
\begin{aligned}
\dot{F}_{v} \leq & \left|\varepsilon_{v}\right| w_{v} h_{v}-k_{7} \varepsilon_{v}^{2}-k_{8}\left|\varepsilon_{v}\right|+b_{4}-\widehat{w}_{v} h_{v}\left|\varepsilon_{v}\right|+b_{5}-\widehat{R}_{v}\left|\varepsilon_{v}\right| \\
& +b_{6}+\left|\varepsilon_{v}\right| R_{v} \leq\left|\varepsilon_{v}\right| \tilde{w}_{v} h_{v}+\left|\varepsilon_{v}\right| \tilde{R}_{v}-k_{7} \varepsilon_{v}^{2}-k_{8}\left|\varepsilon_{v}\right|+b_{4} \\
& +b_{5}+b_{6} .
\end{aligned}
$$


TABle 2: Parameters for the designed control algorithm.

\begin{tabular}{lc}
\hline Section & Parameters \\
\hline Low-pass filter & $\varepsilon_{1}=10, \varepsilon_{2}=10$ \\
Compensation signals & $\vartheta_{1}=10, \vartheta_{2}=0.5$ \\
$\xi_{1}=2, \zeta_{1}=0.01$ & $\xi_{2}=2, \zeta_{2}=0.01$ \\
Auxiliary system & $k_{h}=1.3, k_{h h}=13, k_{1}=0.8, k_{2}=0.2, l_{1}=0.01, \vartheta_{1}=10$, \\
& $\vartheta_{2}=0.5, \vartheta_{3}=10, \vartheta_{4}=0.5, \alpha_{1}=50, r_{1}=0.04, \beta_{1}=10$, \\
Controller & $r_{2}=2, k_{3}=1.5, k_{4}=0.2, k_{5}=2, k_{6}=0.5, \alpha_{3}=0.005$, \\
& $r_{3}=10, \beta_{3}=0.05, r_{4}=0.5, b_{1}=b_{2}=b_{3}=0.001$, \\
& $\xi_{1}=2, \zeta_{1}=0.01, \xi_{2}=2, \zeta_{2}=0.01, \alpha_{v}=0.001, r_{5}=10$, \\
$\beta_{v}=0.001, r_{6}=10, k_{7}=0.6, k_{8}=0.1$, & $b_{4}=b_{5}=b_{6}=0.001$
\end{tabular}

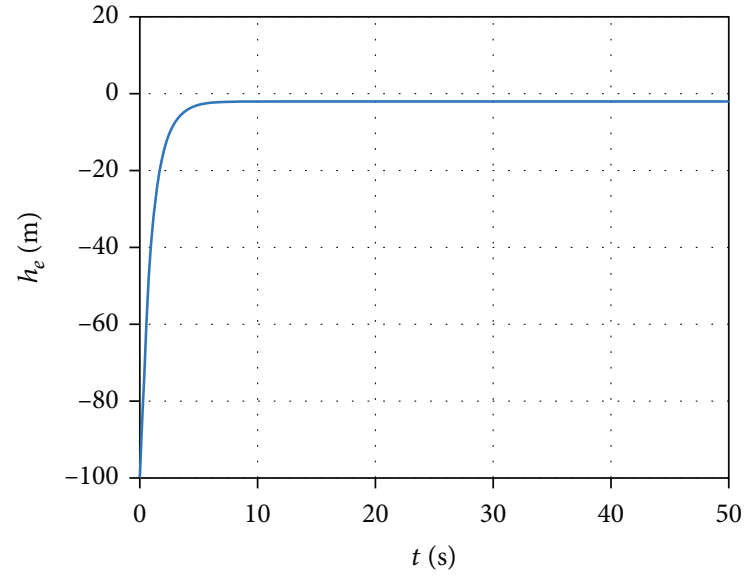

Figure 1: Trajectory tracking error of altitude of GHV.

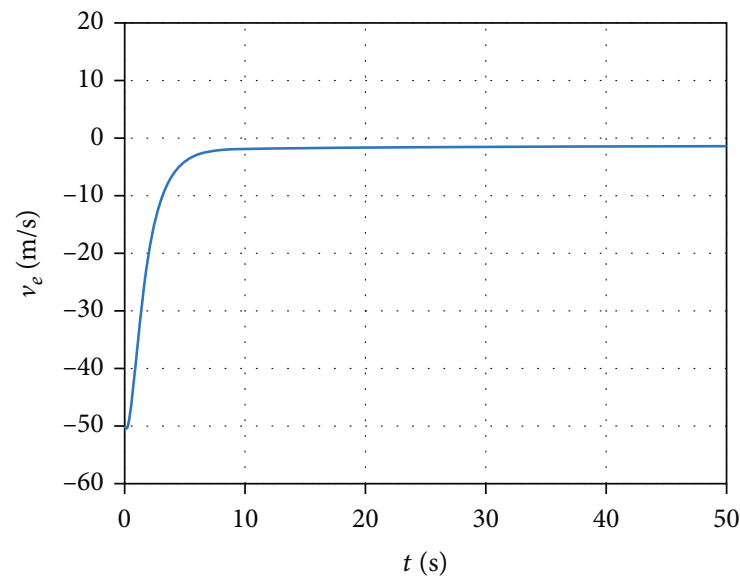

FIGURE 2: Trajectory tracking error of velocity of GHV.

\section{Stability Analysis}

In this section, the stability of the developed controller is demonstrated via Lyapunov-based analysis. It is concluded

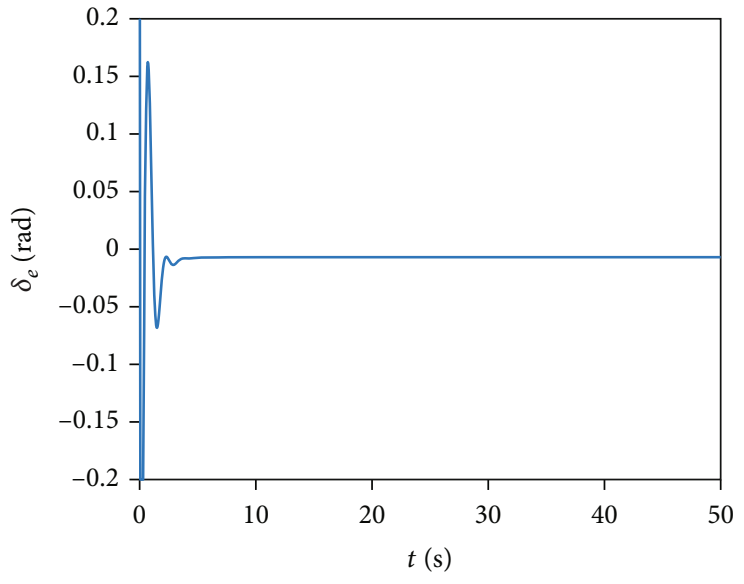

FIgURE 3: The control input of altitude subsystem.

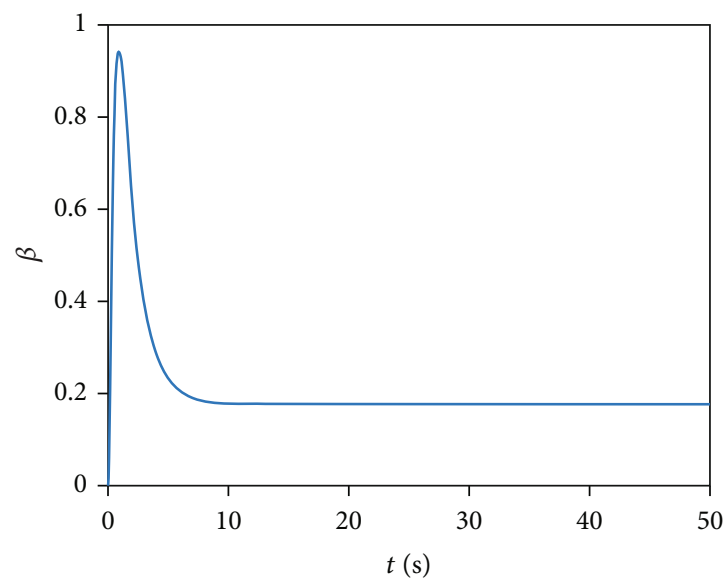

FIGURE 4: The control input of velocity subsystem.

that the control system is capable of maintaining global finite-time convergence. Furthermore, the altitude and velocity tracking errors of GHV will converge to a tiny region containing the origin in finite time. 


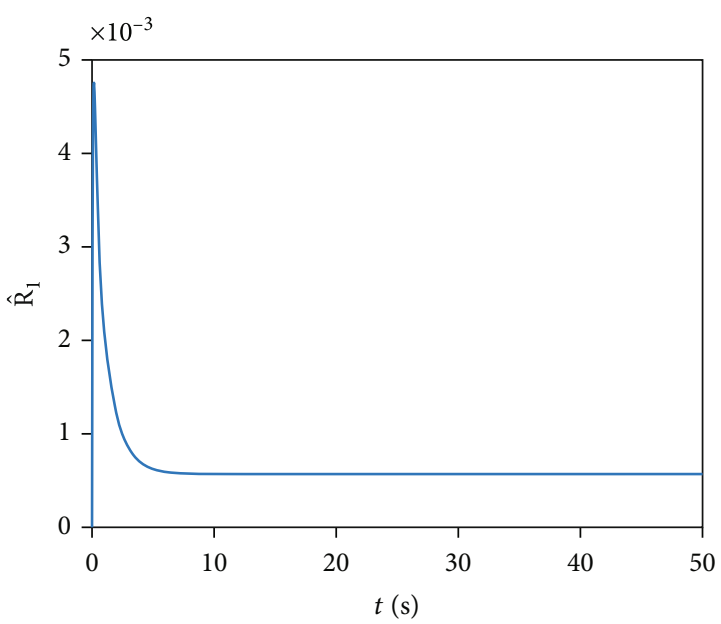

FIgURE 5: The estimation of $\widehat{R}_{1}$.

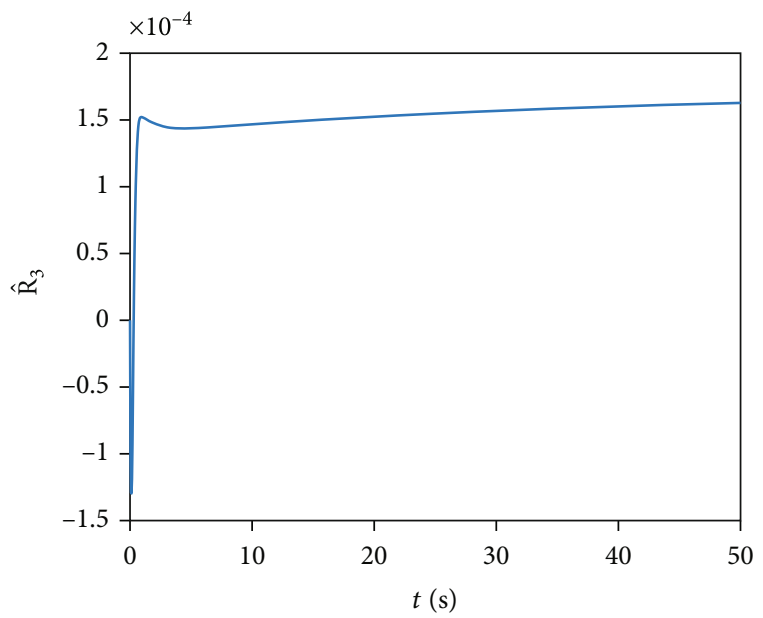

FIgURE 6: The estimation of $\widehat{R}_{3}$.

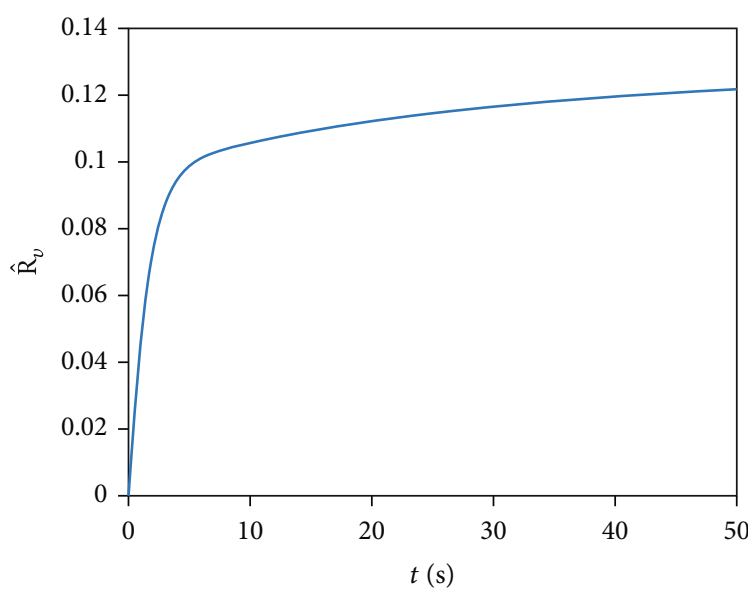

Figure 7: The estimation of $\widehat{R}_{v}$.

Theorem 10. For the GHV dynamics (25) and (57) with Assumptions 3 and 5, the global finite-time convergence is guaranteed under controllers (36), (41), (51), and (66); the adaptive laws (34) and(35), (49) and (50), and (64) and

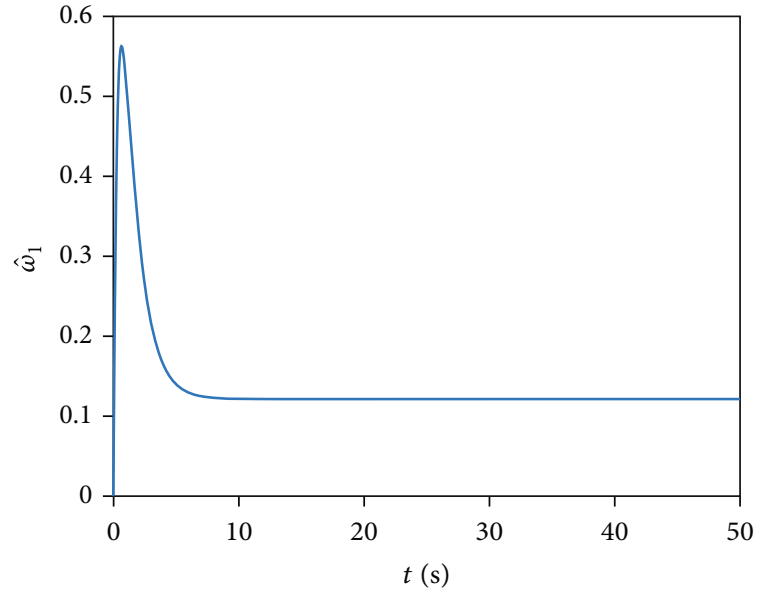

FIgURE 8: The estimation of $\widehat{w}_{1}$.

(65); the compensation signals (33) and (42); and the auxiliary systems (28) and (62).

Proof. The LF is constructed as

$$
\begin{aligned}
F_{4}= & F_{1}+F_{2}+F_{3}+F_{v}+\frac{1}{2 \alpha_{1}} \tilde{w}_{1}^{2}+\frac{1}{2 \alpha_{3}} \tilde{w}_{3}^{2}+\frac{1}{2 \alpha_{v}} \tilde{w}_{v}^{2}+\frac{1}{2 \beta_{1}} \tilde{R}_{1}^{2} \\
& +\frac{1}{2 \beta_{3}} \tilde{R}_{3}^{2}+\frac{1}{2 \beta_{v}} \tilde{R}_{\mathrm{v}}^{2} .
\end{aligned}
$$

Taking the time derivation and substituting above results, it has:

$$
\begin{aligned}
\dot{F}_{4}= & \dot{F}_{1}+\dot{F}_{2}+\dot{F}_{3}+\dot{F}_{v}-\frac{1}{\alpha_{1}} \tilde{w}_{1} \dot{\hat{w}}_{1}-\frac{1}{\alpha_{3}} \tilde{w}_{3} \dot{\hat{w}}_{3}-\frac{1}{\alpha_{v}} \tilde{w}_{v} \dot{\hat{w}}_{v} \\
& -\frac{1}{\beta_{1}} \tilde{R}_{1} \dot{\hat{R}}_{1}-\frac{1}{\beta_{3}} \tilde{R}_{3} \dot{\hat{R}}_{3}-\frac{1}{\beta_{v}} \tilde{R}_{\mathrm{v}} \dot{\hat{R}}_{\mathrm{v}} \leq-\left(k_{1}-1-\bar{g}_{1}\right) \varepsilon_{1}^{2} \\
& -\left(k_{3}-1-\bar{g}_{1}\right) \varepsilon_{2}^{2}-\left(k_{5}-1\right) \varepsilon_{3}^{2}-k_{7} \varepsilon_{v}^{2}-\left(\vartheta_{1}-\frac{1}{4} l_{1}^{2}-\frac{1}{2}\right) \varphi_{1}^{2} \\
& -\left(\vartheta_{3}-\frac{1}{4} l_{2}^{2}-\frac{1}{2}\right) \varphi_{2}^{2}-k_{2}\left|\varepsilon_{1}\right|-k_{4}\left|\varepsilon_{2}\right|-k_{6}\left|\varepsilon_{3}\right|-k_{8}\left|\varepsilon_{v}\right| \\
& -\vartheta_{2}\left|\varphi_{1}\right|-\vartheta_{4}\left|\varphi_{2}\right|+\frac{h_{1}^{2} \widehat{w}_{1}^{2}}{2}+\frac{\widehat{R}_{1}^{2}}{2}+r_{1} \widehat{w}_{1} \tilde{w}_{1}+r_{3} \widehat{w}_{3} \tilde{w}_{3} \\
& +r_{5} \widehat{w}_{v} \tilde{w}_{v}+r_{2} \widehat{R}_{1} \tilde{R}_{1}+r_{4} \widehat{R}_{3} \tilde{R}_{3}+r_{6} \widehat{R}_{v} \tilde{R}_{\mathrm{v}}+\omega^{2}+k_{2} \omega+k_{4} \omega \\
& +\vartheta_{2} \omega+\vartheta_{4} \omega+b_{1}+b_{2}+b_{3}+b_{4}+b_{5}+b_{6} \\
\leq & -\left(k_{1}-1-\bar{g}_{1}\right) \varepsilon_{1}^{2}-\left(k_{3}-1-\bar{g}_{1}\right) \varepsilon_{2}^{2}-\left(k_{5}-1\right) \varepsilon_{3}^{2}-k_{7} \varepsilon_{v}^{2} \\
& -\left(\vartheta_{1}-\frac{1}{4} l_{1}^{2}-\frac{1}{2}\right) \varphi_{1}^{2}-\left(\vartheta_{3}-\frac{1}{4} l_{2}^{2}-\frac{1}{2}\right) \varphi_{2}^{2}-\tilde{w}_{1}^{2}-\tilde{w}_{3}^{2}-\tilde{w}_{v}^{2} \\
& -\tilde{R}_{1}^{2}-\tilde{R}_{3}^{2}-\tilde{R}_{v}^{2}+\tilde{w}_{1}^{2}+\frac{h_{1}^{2} \widehat{w}_{1}^{2}}{2}+r_{1} \widehat{w}_{1} \tilde{w}_{1}+\tilde{w}_{3}^{2}+r_{3} \widehat{w}_{3} \tilde{w}_{3} \\
& +\tilde{w}_{v}^{2}+r_{5} \widehat{w}_{v} \tilde{w}_{v}+\tilde{R}_{1}^{2}+\frac{\widehat{R}_{1}^{2}}{2}+r_{2} \widehat{R}_{1} \tilde{R}_{1}+\tilde{R}_{3}^{2}+r_{4} \widehat{R}_{3} \tilde{R}_{3}+\tilde{R}_{v}^{2} \\
& +r_{6} \widehat{R}_{v} \tilde{R}_{\mathrm{v}}+\omega^{2}+k_{2} \omega+k_{4} \omega+\vartheta_{2} \omega+\vartheta_{4} \omega+b_{1}+b_{2}+b_{3}+b_{4} \\
& +b_{5}+b_{6} \cdot
\end{aligned}
$$




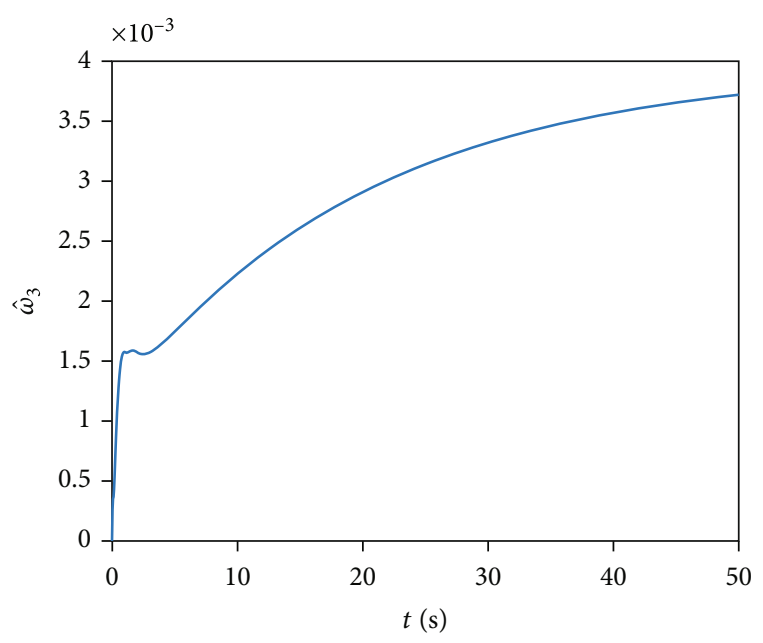

FIGURE 9: The estimation of $\widehat{w}_{3}$.

Take some of the items as quadratic functions to obtain their maximum:

$$
\begin{gathered}
\tilde{w}_{1}^{2}+\frac{h_{1}^{2} \widehat{w}_{1}^{2}}{2}+r_{1} \widehat{w}_{1} \tilde{w}_{1}=\left(w_{1}-w \wedge_{1}\right)^{2}+\frac{h_{1}^{2} \widehat{w}_{1}^{2}}{2}+r_{1} \widehat{w}_{1}\left(w_{1}-\widehat{w}_{1}\right) \\
=-\left(r_{1}-1-\frac{h_{1}^{2}}{2}\right) \widehat{w}_{1}^{2}+\left(r_{1}-2\right) w_{1} \widehat{w}_{1} \\
+w_{1}^{2} \leq \frac{\left(r_{1}^{2}-2 h_{1}^{2}\right) w_{1}^{2}}{4 r_{1}-4-2 h_{1}^{2}}, \\
\tilde{w}_{3}^{2}+r_{3} \widehat{w}_{3} \tilde{w}_{3}=\left(w_{3}-w \wedge_{3}\right)^{2}+r_{3} \widehat{w}_{3}\left(w_{3}-\widehat{w}_{3}\right) \\
=-\left(r_{3}-1\right) \widehat{w}_{3}^{2}+\left(r_{3}-2\right) w_{3} \widehat{w}_{3}+w_{3}^{2} \leq \frac{r_{3}^{2} w_{3}^{2}}{4\left(r_{3}-1\right)}, \\
\tilde{w}_{v}^{2}+r_{5} \widehat{w}_{v} \tilde{w}_{v} \leq \frac{r_{5}^{2} w_{v}^{2}}{4\left(r_{5}-1\right)}, \tilde{R}_{1}^{2}+\frac{\widehat{R}_{1}^{2}}{2}+r_{2} \widehat{R}_{1} \tilde{R}_{1} \leq \frac{\left(r_{2}^{2}-2\right) R_{1}^{2}}{4 r_{2}-6}, \\
\tilde{R}_{3}^{2}+r_{4} \widehat{R}_{3} \tilde{R}_{3} \leq \frac{r_{4}^{2} R_{3}^{2}}{4\left(r_{4}-1\right)}, \tilde{R}_{v}^{2}+r_{6} \widehat{R}_{v} \tilde{R}_{v} \leq \frac{r_{6}^{2} R_{v}^{2}}{4\left(r_{6}-1\right)} .
\end{gathered}
$$

With the substitution of these results, one has

$$
\begin{aligned}
\dot{F}_{4} \leq & -\left(k_{1}-1-\bar{g}_{1}\right) \varepsilon_{1}^{2}-\left(k_{3}-1-\bar{g}_{1}\right) \varepsilon_{2}^{2}-\left(k_{5}-1\right) \varepsilon_{3}^{2}-k_{7} \varepsilon_{v}^{2} \\
& -\left(\vartheta_{1}-\frac{1}{4} l_{1}^{2}-\frac{1}{2}\right) \varphi_{1}^{2}-\left(\vartheta_{3}-\frac{1}{4} l_{2}^{2}-\frac{1}{2}\right) \varphi_{2}^{2}-\tilde{w}_{1}^{2}-\tilde{w}_{3}^{2} \\
& -\tilde{w}_{v}^{2}-\tilde{R}_{1}^{2}-\tilde{R}_{3}^{2}-\tilde{R}_{v}^{2}+\frac{\left(r_{1}^{2}-2 h_{1}^{2}\right) w_{1}^{2}}{4 r_{1}-4-2 h_{1}^{2}}+\frac{r_{3}^{2} w_{3}^{2}}{4\left(r_{3}-1\right)} \\
& +\frac{r_{5}^{2} w_{v}^{2}}{4\left(r_{5}-1\right)}+\frac{\left(r_{2}^{2}-2\right) R_{1}^{2}}{4 r_{2}-6}+\frac{r_{4}^{2} R_{3}^{2}}{4\left(r_{4}-1\right)}+\frac{r_{6}^{2} R_{v}^{2}}{4\left(r_{6}-1\right)}+\omega^{2} \\
& +k_{2} \omega+k_{4} \omega+\vartheta_{2} \omega+\vartheta_{4} \omega+b_{1}+b_{2}+b_{3}+b_{4}+b_{5}+b_{6} \\
\leq & -\kappa_{1} F_{4}+\Delta_{1},
\end{aligned}
$$

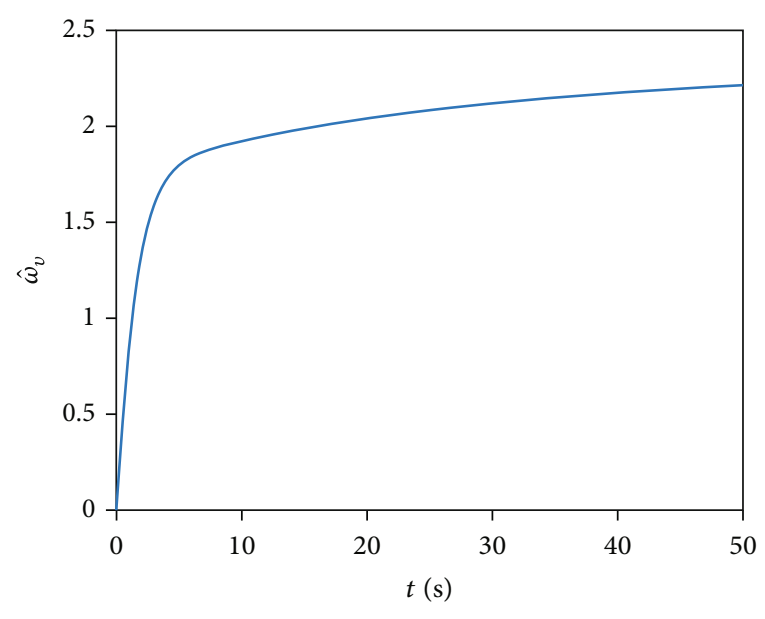

Figure 10: The estimation of $\widehat{w}_{v}$.

where

$$
\begin{aligned}
\kappa_{1}= & \min \left\{2\left(k_{1}-1-\bar{g}_{1}\right), 2\left(k_{3}-1-\bar{g}_{1}\right), 2\left(k_{5}-1\right), 2 k_{7},\left(2 \vartheta_{1}-\frac{1}{2} l_{1}^{2}-1\right),\right. \\
& \left.\cdot\left(2 \vartheta_{3}-\frac{1}{2} l_{2}^{2}-1\right), 2 \alpha_{1}, 2 \alpha_{3}, 2 \alpha_{v}, 2 \beta_{1}, 2 \beta_{3}, 2 \beta_{v}\right\}, \\
\Delta_{1}= & \frac{\left(r_{1}^{2}-2 h_{1}^{2}\right) w_{1}^{2}}{4 r_{1}-4-2 h_{1}^{2}}+\frac{r_{3}^{2} w_{3}^{2}}{4\left(r_{3}-1\right)}+\frac{r_{5}^{2} w_{v}^{2}}{4\left(r_{5}-1\right)}+\frac{\left(r_{2}^{2}-2\right) R_{1}^{2}}{4 r_{2}-6} \\
& +\frac{r_{4}^{2} R_{3}^{2}}{4\left(r_{4}-1\right)}+\frac{r_{6}^{2} R_{v}^{2}}{4\left(r_{6}-1\right)}+\omega^{2}+k_{2} \omega+k_{4} \omega+\vartheta_{2} \varpi+\vartheta_{4} \varpi \\
& +b_{1}+b_{2}+b_{3}+b_{4}+b_{5}+b_{6} .
\end{aligned}
$$

The result indicates that the designed controller is asymptotically stable. Therefore, as long as the initial conditions are bounded, the estimation errors must have uniform ultimate boundedness. Thus, there exist constants $\bar{w}_{i}, i=1$, $3, v$, satisfying $\bar{w}_{i}>\widehat{w}_{i}$ and $\bar{w}_{i}>w_{i} . \bar{R}_{i}, i=1,3, v$, follow the same principle, i.e.,

$$
\begin{gathered}
\bar{w}_{i}-\widehat{w}_{i}>w_{i}-\widehat{w}_{i}=\tilde{w}_{i}, \quad i=1,3, v, \\
\bar{R}_{i}-\widehat{R}_{i}>R_{i}-\widehat{R}_{i}=\tilde{R}_{i}, \quad i=1,3, v .
\end{gathered}
$$

Rebuild the LF as

$$
\begin{aligned}
F_{5}= & F_{1}+F_{2}+F_{3}+F_{v}+\frac{1}{\alpha_{1}}\left(\bar{w}_{1}-w \wedge_{1}\right)^{2}+\frac{1}{\alpha_{3}}\left(\bar{w}_{3}-w \wedge_{3}\right)^{2} \\
& +\frac{1}{\alpha_{v}}\left(\bar{w}_{v}-w \wedge_{v}\right)^{2}+\frac{1}{\beta_{1}}\left(\bar{R}_{1}-R \wedge_{1}\right)^{2}+\frac{1}{\beta_{3}}\left(\bar{R}_{3}-R \wedge_{3}\right)^{2} \\
& +\frac{1}{\beta_{v}}\left(\bar{R}_{v}-R \wedge_{v}\right)^{2} .
\end{aligned}
$$


Differentiating $F_{5}$ along Equation (80) yields

$$
\begin{aligned}
& \dot{F}_{5}=\dot{F}_{1}+\dot{F}_{2}+\dot{F}_{3}+\dot{F}_{v}-\frac{2}{\alpha_{1}}\left(\bar{w}_{1}-\widehat{w}_{1}\right) \dot{\hat{w}}_{1}-\frac{2}{\alpha_{3}}\left(\bar{w}_{3}-\widehat{w}_{3}\right) \dot{\hat{w}}_{3} \\
& -\frac{2}{\alpha_{v}}\left(\bar{w}_{v}-\widehat{w}_{v}\right) \dot{\hat{w}}_{v}-\frac{2}{\beta_{1}}\left(\bar{R}_{1}-\widehat{R}_{1}\right) \dot{\hat{R}}_{1}-\frac{2}{\beta_{3}}\left(\bar{R}_{3}-\widehat{R}_{3}\right) \dot{\hat{R}}_{3} \\
& -\frac{2}{\beta_{v}}\left(\bar{R}_{v}-\widehat{R}_{v}\right) \dot{\hat{R}}_{v} \leq-\left(k_{1}-1-\bar{g}_{1}\right) \varepsilon_{1}^{2}-\left(k_{3}-1-\bar{g}_{1}\right) \varepsilon_{2}^{2} \\
& -\left(k_{5}-1\right) \varepsilon_{3}^{2}-k_{7} \varepsilon_{v}^{2}-\left(\vartheta_{1}-\frac{1}{4} l_{1}^{2}-\frac{1}{2}\right) \varphi_{1}^{2} \\
& -\left(\vartheta_{3}-\frac{1}{4} l_{2}^{2}-\frac{1}{2}\right) \varphi_{2}^{2}-k_{2}\left|\varepsilon_{1}\right|-k_{4}\left|\varepsilon_{2}\right|-k_{6}\left|\varepsilon_{3}\right|-k_{8}\left|\varepsilon_{v}\right| \\
& -\vartheta_{2}\left|\varphi_{1}\right|-\vartheta_{4}\left|\varphi_{2}\right|-\left|\varepsilon_{1}\right| h_{1}\left(\bar{w}_{1}-\widehat{w}_{1}\right)-\left|\varepsilon_{3}\right| h_{3}\left(\bar{w}_{3}-\widehat{w}_{3}\right) \\
& -\left|\varepsilon_{v}\right| h_{v}\left(\bar{w}_{v}-\widehat{w}_{v}\right)-\left|\varepsilon_{1}\right|\left(\bar{R}_{1}-\widehat{R}_{1}\right)-\left|\varepsilon_{3}\right|\left(\bar{R}_{3}-\widehat{R}_{3}\right) \\
& -\left|\varepsilon_{v}\right|\left(\bar{R}_{v}-\widehat{R}_{v}\right)+2 r_{1}\left(\bar{w}_{1}-\widehat{w}_{1}\right) \widehat{w}_{1}+2 r_{3}\left(\bar{w}_{3}-\widehat{w}_{3}\right) \widehat{w}_{3} \\
& +2 r_{5}\left(\bar{w}_{v}-\widehat{w}_{v}\right) \widehat{w}_{v}+2 r_{2}\left(\bar{R}_{1}-\widehat{R}_{1}\right) \widehat{R}_{1}+2 r_{4}\left(\bar{R}_{3}-\widehat{R}_{3}\right) \widehat{R}_{3} \\
& +2 r_{6}\left(\bar{R}_{v}-\widehat{R}_{v}\right) \widehat{R}_{v}+\frac{h_{1}^{2} \widehat{w}_{1}^{2}}{2}+\frac{\widehat{R}_{1}^{2}}{2}+\omega^{2}+k_{2} \omega+k_{4} \omega \\
& +\vartheta_{2} \varpi+\vartheta_{4} \varpi+b_{1}+b_{2}+b_{3}+b_{4}+b_{5}+b_{6} \text {. }
\end{aligned}
$$

In particular, the results hold as

$2 r_{1}\left(\bar{w}_{1}-\widehat{w}_{1}\right) \widehat{w}_{1}+\frac{h_{1}^{2} \widehat{w}_{1}^{2}}{2}=-\left(2 r_{1}-\frac{h_{1}^{2}}{2}\right) \widehat{w}_{1}^{2}+2 r_{1} \bar{w}_{1} \widehat{w}_{1} \leq \frac{2 r_{1}^{2} \bar{w}_{1}^{2}}{4 r_{1}-h_{1}^{2}}$, where

$$
\begin{gathered}
2 r_{3}\left(\bar{w}_{3}-\widehat{w}_{3}\right) \widehat{w}_{3}=-2 r_{3} \widehat{w}_{3}^{2}+2 r_{3} \bar{w}_{3} \widehat{w}_{3} \leq \frac{r_{3} \bar{w}_{3}^{2}}{2}, \\
2 r_{5}\left(\bar{w}_{v}-\widehat{w}_{v}\right) \widehat{w}_{v} \leq \frac{r_{5} \bar{w}_{v}^{2}}{2}, 2 r_{2}\left(\bar{R}_{1}-\widehat{R}_{1}\right) \widehat{R}_{1}+\frac{\widehat{R}_{1}^{2}}{2} \leq \frac{2 r_{2}^{2} \bar{R}_{1}^{2}}{4 r_{2}-1}, \\
2 r_{4}\left(\bar{R}_{3}-\widehat{R}_{3}\right) \widehat{R}_{3} \leq \frac{r_{4} \bar{R}_{3}^{2}}{2}, 2 r_{6}\left(\bar{R}_{v}-\widehat{R}_{v}\right) \widehat{R}_{v} \leq \frac{r_{6} \bar{R}_{v}^{2}}{2} .
\end{gathered}
$$

Substituting these results into Equation (81), it leads to

$$
\begin{aligned}
\dot{F}_{5} \leq & -k_{2}\left|\varepsilon_{1}\right|-k_{4}\left|\varepsilon_{2}\right|-k_{6}\left|\varepsilon_{3}\right|-k_{8}\left|\varepsilon_{v}\right|-\vartheta_{2}\left|\varphi_{1}\right|-\vartheta_{4}\left|\varphi_{2}\right| \\
& -\left|\varepsilon_{1}\right| h_{1}\left(\bar{w}_{1}-\widehat{w}_{1}\right)-\left|\varepsilon_{3}\right| h_{3}\left(\bar{w}_{3}-\widehat{w}_{3}\right)-\left|\varepsilon_{v}\right| h_{v}\left(\bar{w}_{v}-\widehat{w}_{v}\right) \\
& -\left|\varepsilon_{1}\right|\left(\bar{R}_{1}-\widehat{R}_{1}\right)-\left|\varepsilon_{3}\right|\left(\bar{R}_{3}-\widehat{R}_{3}\right)-\left|\varepsilon_{v}\right|\left(\bar{R}_{v}-\widehat{R}_{v}\right) \\
& +\frac{2 r_{1}^{2} \bar{w}_{1}^{2}}{4 r_{1}-h_{1}^{2}}+\frac{r_{3} \bar{w}_{3}^{2}}{2}+\frac{r_{5} \bar{w}_{v}^{2}}{2}+\frac{2 r_{2}^{2} \bar{R}_{1}^{2}}{4 r_{2}-1}+\frac{r_{4} \bar{R}_{3}^{2}}{2}+\frac{r_{6} \bar{R}_{v}^{2}}{2} \\
& +\omega^{2}+k_{2} \omega+k_{4} \oplus+\vartheta_{2} \omega+\vartheta_{4} \varpi+b_{1}+b_{2}+b_{3}+b_{4}+b_{5} \\
& +b_{6} \leq-\kappa_{2} F_{5}+\Delta_{2},
\end{aligned}
$$

$$
\begin{gathered}
\kappa_{2}=\min \left\{\sqrt{2} k_{2}, \sqrt{2} k_{4}, \sqrt{2} k_{6}, \sqrt{2} k_{8}, \sqrt{2} \vartheta_{2}, \sqrt{2} \vartheta_{4}, \sqrt{\alpha_{1}}\left|\varepsilon_{1}\right| h_{1}, \sqrt{\alpha_{3}}\left|\varepsilon_{3}\right| h_{3}, \sqrt{\alpha_{v}}\left|\varepsilon_{v}\right| h_{v}, \sqrt{\beta_{1}}\left|\varepsilon_{1}\right|, \sqrt{\beta_{3}}\left|\varepsilon_{3}\right|, \sqrt{\beta_{v}}\left|\varepsilon_{v}\right|\right\}, \\
\Delta_{2}=\frac{2 r_{1}^{2} \bar{w}_{1}^{2}}{4 r_{1}-h_{1}^{2}}+\frac{r_{3} \bar{w}_{3}^{2}}{2}+\frac{r_{5} \bar{w}_{v}^{2}}{2}+\frac{2 r_{2}^{2} \bar{R}_{1}^{2}}{4 r_{2}-1}+\frac{r_{4} \bar{R}_{3}^{2}}{2}+\frac{r_{6} \bar{R}_{v}^{2}}{2}+\omega^{2}+k_{2} \emptyset+k_{4} \emptyset+\vartheta_{2} \emptyset+\vartheta_{4} \emptyset+b_{1}+b_{2}+b_{3}+b_{4}+b_{5}+b_{6} .
\end{gathered}
$$

As a result, it is indicated that all the tracking errors and estimation errors converge into a tiny region in finite time. The proof of Theorem 10 is completed.

Remark 11. Consequently, it is necessary to make a guiding explanation for the selection of controller parameters from a theoretical perspective. Considering Equations (83), (84) and (85), the overall feedback gain depends on $k_{i}$, which indicates that the larger $k_{i}$ leads to the faster stabilization rate, and accordingly, the required control torque will increase. On the other hand, the design parameters $b_{i}$ play a significant role in steady-state error. Obviously, if the value of $b_{i}$ is small, the steady-state error of the closed-loop system will decrease, while the chattering phenomenon of the actuator will increase. Therefore, the best choice is to compromise the two indicators and determine the values of these parameters, so that the controller possesses a satisfactory overall performance.

\section{Numerical Simulations}

In this section, a simulation example is provided to demonstrate the stability and effectiveness of the designed controller. According to the dynamics (1), (2), (3), (4), (5), (6), (7), (8), (9), (10), (11), (12), (13), (14), (15), (16) and (17) of GHV, the initial values and desired trajectories are given to facilitate the implementation of the tracking controller, while various performances will be simultaneously recorded and exhibited. Necessarily, the initial values of system states are set as Table 1, and the designed parameters of the control algorithm are shown in Table 2 . 
Subsequently, to test the trajectory tracking performance and robustness against disturbance, the reference altitude and velocity signals, the lumped external disturbances, and the saturation constraint of actuator are defined as

$$
\begin{gathered}
V_{d}=15110, \\
h_{d}=110100, \\
d_{1}=0.01 * \sin (0.1 * t), \\
d_{2}=0.01 * \cos (0.1 * t), \\
u_{1 m}=0.2 \mathrm{rad}, \\
u_{2 m}=0.9 .
\end{gathered}
$$

Consequently, the main results are given in Figures 1-10.

To start with, curves of trajectory tracking errors are given in Figures 1 and 2, which indicate that the altitude and velocity tracking missions are completed within $10 \mathrm{~s}$. Undoubtedly, the finite-time controller shows great superiority in contrast with ordinary asymptotic convergence. Afterwards, the feedback input signals of the closed-loop system are shown in Figures 3 and 4 . Practically speaking, the altitude control torque and velocity control torque converges into a tiny region containing origin within $10 \mathrm{~s}$. It is noteworthy that the saturation nonlinearity of actuators is well accommodated during the control process. What is more, Figures 5-7 give the curves of estimated values $\widehat{R}_{1}, \widehat{R}_{3}$, and $\widehat{R}_{v}$, which converge to a constant in finite time. The adaptive parameters of RBFNNs are addressed in Figures 8-10. Obviously, estimations of the norm of the weight matrix will always remain bounded under the proposed method. To conclude, performances of the proposed controller satisfy the design requirements.

\section{Conclusion}

This paper investigates the trajectory tracking control of GHVs exposed to system uncertainties and input saturation constraints. On the basis of the longitudinal model of GHVs, the command filter-based adaptive neural controller is constructed to guarantee the finite-time stability of the closedloop system. Specially, considering the defect of EOC associated with a conservative backstepping design, the low-pass filters are introduced and compensation signals are designed to avoid the repeat derivation on virtual command. Meanwhile, the MLP algorithm-based function approximation is utilized to cope with the inaccurate model information, in which the tedious analytical computation is simplified significantly. The input nonlinearity caused by actuator saturation is addressed via a modified auxiliary system. Finally, the rigorous mathematical analysis and simulation results are provided to confirm the validity of the proposed architecture.

\section{Data Availability}

No data were used to support to this study.

\section{Conflicts of Interest}

The authors declare that there are no conflicts of interest regarding publication of this article.

\section{Acknowledgments}

This work is cosupported by the Defense Industrial Technology Development Program (JCKY2016205C013) and Aeronautical Science Foundation of China (2015ZC18005).

\section{References}

[1] Z. Ping, J. Ju, Y. Chaojun, and W. Chengyu, "Neuro-adaptive sliding mode fault-tolerant control of hypersonic vehicle under actuator fault," in 2019 Chinese Control And Decision Conference (CCDC), pp. 1558-1563, Nanchang, China, 2019.

[2] Y. Cheng, S. Yang, B. Xu, D. Zhang, and Y. Zhang, "Discrete sliding mode adaptive control of GHV with prediction model," IEEE 28th International Symposium on Industrial Electronics (ISIE), 2019, pp. 2253-2258, Vancouver, BC, Canada, 2019.

[3] Y. Wu, F. Ma, X. Liu, Y. Hua, X. Liu, and G. Li, "Super twisting disturbance observer-based fixed-time sliding mode backstepping control for air-breathing hypersonic vehicle," IEEE Access, vol. 8, pp. 17567-17583, 2020.

[4] X. Cheng, P. Wang, and G. Tang, "Adaptive back-stepping control for flexible hypersonic vehicle using ESO," in 2018 9th International Conference on Mechanical and Aerospace Engineering (ICMAE),, pp. 617-622, Budapest, Hungary, July 2018.

[5] C. Li, J. Sun, and Y. Guo, "Adaptive back-stepping tracking control of the hypersonic vehicle with input saturation," in 2019 IEEE 4th Advanced Information Technology, Electronic and Automation Control Conference (IAEAC), pp. 839-845, Chengdu, China, 2019.

[6] L. Dou, P. Su, Q. Zong, and Z. Ding, "Fuzzy disturbance observer-based dynamic surface control for air-breathing hypersonic vehicle with variable geometry inlets," IET Control Theory \& Applications, vol. 12, no. 1, pp. 10-19, 2018.

[7] X. Bu, D. Wei, and G. He, "A robust constrained control approach for flexible air-breathing hypersonic vehicles," International Journal of Robust and Nonlinear Control, vol. 30, no. 7, pp. 2752-2776, 2020.

[8] X. Bu, X. Yu, and H. Lei, "An adaptive critic design-based fuzzy neural controller for hypersonic vehicles: predefined behavioral nonaffine control," IEEE-ASME Transactions on Mechatronics, vol. 24, no. 4, pp. 1871-1881, 2019.

[9] Z. Wu, J. Lu, Q. Zhou, and J. Shi, "Modified adaptive neural dynamic surface control for morphing aircraft with input and output constraints," Nonlinear Dynamics, vol. 87, no. 4, pp. 2367-2383, 2017.

[10] H. Chi and F. Yu, "Compound control for hypersonic vehicles based on dynamic compensation," Chinese automation congress (CAC), 2018, pp. 3523-3528, Xi'an, China, 2018.

[11] S. Zhao and X. Li, "Prescribed performance fault tolerant control for hypersonic flight vehicles with actuator failures," IEEE Access, vol. 7, pp. 100187-100204, 2019.

[12] Y. Chen, Y. Wei, G. R. Duan, and L. G. Tan, "Robust finite time tracking control of flexible hypersonic vehicle with uncertainties," in 2020 IEEE 4th Information Technology, Networking, Electronic and Automation Control Conference (ITNEC), pp. 233-239, Chongqing, China, June 2020. 
[13] X. Zhang, K. Chen, and W. Fu, "Fuzzy adaptive control for generic hypersonic vehicles with non-affine aerodynamic characteristics and output constraints," in 2019 19th International Conference on Control, Automation and Systems (ICCAS), pp. 71-75, Jeju, Republic of Korea, 2019.

[14] Y. Liu, J. Yi, and Z. Pu, "Desired compensation based adaptive fuzzy control for hypersonic vehicle with measurement noises," in 2018 IEEE International Conference on Fuzzy Systems (FUZZ-IEEE),, pp. 1-7, Rio de Janeiro, Brazil, 2018.

[15] X. Li and G. Li, "Novel fuzzy approximation control scheme for flexible air-breathing hypersonic vehicles with non-affine dynamics and amplitude and rate constraints," IEEE Access, vol. 7, pp. 73602-73616, 2019.

[16] Y. Ma and Y. Cai, "A fuzzy model predictive control based upon adaptive neural network disturbance observer for a constrained hypersonic vehicle," IEEE Access, vol. 6, pp. 59275938, 2018.

[17] X. Bu, "Air-breathing hypersonic vehicles funnel control using neural approximation of non-affine dynamics," IEEE/ASME Transactions on Mechatronics, vol. 23, no. 5, pp. 2099-2108, 2018.

[18] J. Wang, J. Wang, P. Yang, K. Liu, X. Yu, and J. Hu, “Adaptive sliding mode control for a flexible hypersonic vehicle with actuator saturation," in 2016 35th Chinese Control Conference (CCC), pp. 647-651, Chengdu, China, July 2016.

[19] G. Jianguo, L. Yifei, and Z. Jun, "Finite time control for generic hypersonic vehicle," in 2018 Chinese Control And Decision Conference (CCDC), pp. 3727-3731, Shenyang, China, June 2018.

[20] X. Yu, P. Li, and Y. Zhang, "The design of fixed-time observer and finite-time fault-tolerant control for hypersonic gliding vehicles," IEEE Transactions on Industrial Electronics, vol. 65, no. 5, pp. 4135-4144, 2018.

[21] S. Jing-guang and S. Shen-min, "Tracking control of hypersonic vehicle with input saturation based on adaptive backstepping method," in 2017 36th Chinese Control Conference (CCC), pp. 622-628, Dalian, China, July 2017.

[22] H. Sun, S. Li, and C. Sun, "Adaptive fault-tolerant controller design for airbreathing hypersonic vehicle with input saturation," Journal of Systems Engineering and Electronics, vol. 24, no. 3, pp. 488-499, 2013.

[23] K. Esfandiari, F. Abdollahi, and H. A. Talebi, "Adaptive control of uncertain nonaffine nonlinear systems with input saturation using neural networks," IEEE Transactions on Neural Networks and Learning Systems, vol. 26, no. 10, pp. 23112322, 2015.

[24] J. T. Parker, A. Serrani, S. Yurkovich, M. A. Bolender, and D. B. Doman, "Control-oriented modeling of an air-breathing hypersonic vehicle," Journal of Guidance, Control, and Dynamics, vol. 30, no. 3, pp. 856-869, 2007.

[25] B. Xu, "Robust adaptive neural control of flexible hypersonic flight vehicle with dead-zone input nonlinearity," Nonlinear Dynamics, vol. 80, no. 3, pp. 1509-1520, 2015.

[26] R. J. Wallsgrove and M. R. Akella, "Globally stabilizing saturated attitude control in the presence of bounded unknown disturbances," Journal of Guidance, Control, and Dynamics, vol. 28, no. 5, pp. 957-963, 2005. 